\title{
Exhaled NO may predict the decline in lung function in bronchiolitis obliterans syndrome
}

\author{
O. Brugière*, G. Thabut*, H. Mal*, A. Marceau*, G. Dauriat*, R. Marrash-Chahla*, \\ Y. Castier ${ }^{\#}$, G. Lesèche ${ }^{\#}$, M. Colombat and M. Fournier*
}

ABSTRACT: Bronchiolitis obliterans syndrome (BOS) remains the leading cause of morbidity/ mortality following lung transplantation. In recipients with BOS, markers predicting the decline in lung function are needed. The aim of this longitudinal study was to determine whether exhaled nitric oxide fraction $(F \mathrm{eNO})$ measurements provide useful information for discriminating patients with unstable BOS from those with stable BOS.

During a 14-month period, 145 FeNO measurements were performed in 50 lung transplant recipients. Among them, 16 recipients with BOS (32 FeNO measurements) were analysed. For each FeNO measurement, the patients were classified into three groups according to the decline in forced expiratory volume in one second (FEV1) within the following 6 months: 1) stable BOS free; 2) stable BOS (decline in FEV 1 of $<5 \%$ ); and 3) unstable BOS (decline in FEV 1 of $\geqslant 15 \%$ ).

The mean FeNO in patients with unstable BOS was significantly increased compared with that in stable BOS-free patients (18.4 \pm 5.7 versus $9.7 \pm 3.7 \mathrm{ppb})$ and that in patients with stable BOS (18.4 \pm 5.7 versus $9.7 \pm 3.3 \mathrm{ppb})$.

The present findings suggest that, in patients with bronchiolitis obliterans syndrome, a raised exhaled nitric oxide fraction may predict the development of worrisome functional impairment during long-term follow-up.

KEYWORDS: Bronchiolitis obliterans syndrome, exhaled nitric oxide, lung transplantation

$\mathbf{A}$ lthough lung transplantation is now an established treatment for patients with end-stage lung disease, the long-term functional status and survival of lung transplant recipients are still limited by the occurrence of bronchiolitis obliterans syndrome (BOS). BOS has been defined as a clinical syndrome of irreversible progressive airway obstruction in the pulmonary allograft caused by the presence of obliterative bronchiolitis. It is thought to represent a form of chronic rejection, which develops in $>50 \%$ of lung transplant recipients 3 yrs after transplantation [1] and is the commonest cause of late graft failure. Early detection and treatment of BOS seem to be the most important factors that may permit stabilisation of lung function in patients with BOS, since better survival has been reported in patients with lower BOS stages [2]. In addition, once the BOS is established, another clinical challenge is to determine an adequate level of immunosuppression, allowing stability of lung function without excessive risk of infection due to overt immunosuppression. In this regard, there is no marker that can predict the different functional patterns of BOS over time. These patterns include either rapid loss of lung function, slow progression or intermittent loss of function, with a long plateau period during which pulmonary function is stable [3,4]. Therefore, there is a need for new tools to monitor the lung allograft, especially for immunological complications.

Nitric oxide (NO) is implicated in the pathophysiology of airway diseases [5]. The upregulation of the inducible form of NO synthase (iNOS) in airway epithelial cells is associated with increased production and prolonged release of NO [6]. The fraction of $\mathrm{NO}$ in exhaled breath (FeNO) has been shown to be correlated with the levels of $\mathrm{NO}$ found in the lower respiratory tract [7]. Compared with healthy subjects, FeNO is increased in diseases associated with airway inflammation, such as asthma or respiratory infection $[8,9]$. It has also been found that higher $\mathrm{NO}$ levels in patients with fibrosing lung disease were associated with increased disease activity [10] or subclinical inflammation [11], and that the same patients with more advanced fibrosing lung disease exhibited normal NO levels [11].
AFFILIATIONS

Depts of *Pneumology,

\#Thoracic Surgery, and

"Pathology, Beaujon Hospital,

Clichy, France.

CORRESPONDENCE

0 . Brugière

Service de Pneumologie et

Réanimation Respiratoire

Hôpital Beaujon

100 bd du Gén. Leclerc

92000 Clichy

France

Fax: 33142708719

E-mail: olivier.brugiere@bjn.

ap-hop-paris.fr

Received:

May 122004

Accepted after revision:

December 102004

SUPPORT STATEMENT

This study was supported by the Comité National contre les Maladies Respiratoires (Paris, France). 
Accordingly, there is increasing interest in FeNO as a noninvasive marker of airway inflammation after lung transplantation. In particular, it has recently been demonstrated that lung transplant recipients with BOS exhibit increased FeNO compared with stable lung transplantation patients and healthy nonsmoking controls [12-14], and that FeNO may be helpful in the early diagnosis of BOS [15].

As suggested for fibrosing lung disease [10, 11], it was hypothesised in the present study that the inflammatory activity in the airways of patients with unstable BOS would lead to increased iNOS expression and a subsequent increase in FeNO, which would be downregulated once the inflammatory phase is replaced by fibrosis in patients with stable BOS. In this regard, the clinical utility of FeNO measurements was evaluated in lung transplant recipients with BOS followed-up in the Beaujon Hospital (Clichy, France) over a 14-month period. The aim of the present clinical study was to determine whether FeNO measurements provide information that is useful for discriminating patients with unstable BOS from those with stable BOS.

\section{METHODS}

\section{Study population}

Between November 2001 and December 2002, all lung transplant recipients surviving beyond 6 months following transplantation were eligible for recording of FeNO. FeNO measurement was performed: 1) in ambulatory recipients at each routine follow-up, and 2) in the case of respiratory complication.

During the study period, FeNO was measured in 50 patients (11 double-lung (bilateral lung transplantation (BLT)) and 39 single-lung (single lung transplantation (SLT)) transplant recipients), at a median time after transplantation of 600 days (range 180-4,400 days). The median number of FeNO measurements was three per patient (range 1-6). Each patient was followed-up for $\geqslant 6$ months after the last FeNO measurement. The pre-transplant diagnoses were emphysema with or without $\alpha_{1}$-antitrypsin deficiency $(n=22)$, idiopathic pulmonary fibrosis $(n=13)$, cystic fibrosis and other bronchiectatic diseases $(n=5)$, and other respiratory diseases $(n=10)$.

\section{Immunosuppression}

The immunosuppressive regimen consisted of cyclosporin A (trough level 200-300 ng. $\mathrm{mL}^{-1}$ for the first 3 months, and thereafter according to time after lung transplantation and renal function), azathioprine $\left(1-2 \mathrm{mg} \cdot \mathrm{kg}^{-1} \cdot \mathrm{day}^{-1}\right)$ and prednisone $\left(\geqslant 7.5 \mathrm{mg} \cdot \mathrm{day}^{-1}\right)$. Intravenous methylprednisolone was given at a dose of $500 \mathrm{mg}$ pre-operatively and before reperfusion of the graft, $0.5 \mathrm{mg} \cdot \mathrm{kg}^{-1} \cdot \mathrm{day}^{-1}$ on the following days, and thereafter replaced by oral prednisone, which was progressively tapered to $0.1 \mathrm{mg} \cdot \mathrm{kg}^{-1} \cdot \mathrm{day}^{-1}$ after 12 weeks. Rabbit antithymocyte globulin induction therapy was given during the post-operative period $\left(1.5 \mathrm{mg} \cdot \mathrm{kg}^{-1} \cdot \mathrm{day}^{-1}\right.$ for 3 days $)$ only in patients without cytomegalovirus (CMV) mismatching.

\section{Lung transplantation protocols}

At each routine visit (at least every 3 months), or, in the case of pulmonary complication, pulmonary function testing (forced expiratory volume in one second (FEV1) and vital capacity) was performed in accordance with standardised American
Thoracic Society guidelines $\geqslant 1 \mathrm{~h}$ before the FeNO measurement was recorded. Fibreoptic bronchoscopy with bronchoalveolar lavage (BAL) and transbronchial biopsy (TBB) was performed only for determination of clinical, physiological or radiographic changes. BAL specimens were cultured for determination of the presence of bacteria (including Nocardia, Actinomyces, Legionella and mycobacteria), viruses (with immunofluorescence testing for respiratory viruses and culture for CMV) and fungi (including Aspergillus spp.). All TBB specimens were interpreted by the same pathologist and acute rejection histology was graded according to the system proposed by YOUSEM et al. [16]. An episode of acute rejection was defined after histological confirmation through TBB. Acute rejection was treated with daily infusion of methylprednisolone ( $1 \mathrm{~g}$ intravenously) for 3 days, followed by prednisone $\left(1 \mathrm{mg} \cdot \mathrm{kg}^{-1} \cdot \mathrm{day}^{-1}\right)$, tapered to a maintenance dose over 3 weeks. Resistant acute rejection was treated with rabbit antithymocyte globulin $\left(2.5 \mathrm{mg} \cdot \mathrm{kg}^{-1} \cdot \mathrm{day}^{-1}\right.$ for 5 days $)$. BOS was defined according to the revised classification system of the International Society for Heart and Lung Transplantation [4]. According to this classification, potential BOS (BOS 0-p) was defined by a 10-19\% decrease in FEV1 from baseline [4]. BOS status was ascertained with repeated pulmonary function testing at least every 3 months after transplantation.

\section{Diagnostic categories}

The clinical outcome of the patients was analysed within the 6 months following each FeNO measurement. For each FeNO measurement, the patients were allocated to the following categories by a physician blinded to the FeNO results.

\section{Group 1: stable patients free of BOS}

Group 1 patients were those without any acute pulmonary complication within 1 month before or after FeNO measurement, and without chronic pulmonary complication at the time of any FeNO measurement during the study period.

\section{Group 2: stable BOS}

Group 2 patients were those with established BOS ( $\geqslant$ BOS 1 ) at the time of FeNO measurement, free from any acute complication (acute rejection, lymphocytic bronchiolitis and infection) within the previous month, and who retained stable pulmonary function for $\geqslant 6$ months following the date of FeNO measurement. Pulmonary function was considered stable if the loss in FEV1 was $<5 \%$ within the following 6 months.

\section{Group 3: unstable BOS}

Patients were classified as unstable in the case of: 1) an initial decline in pulmonary function corresponding to the first diagnosis of established BOS (BOS 1) at the date of FeNO measurement, or 2) at least potential BOS (>BOS 0-p) at the time of FeNO measurement with subsequent pulmonary functional decline within the following 3 months, leading to established BOS ( $\geqslant$ BOS 1). Except for one patient (No. 2; table 1), patients classified as having unstable BOS showed a fractional decrease in FEV1 (expressed as the percentage decrease from the highest previous baseline FEV1 not attributed to other causes (exclusion of infection, acute rejection and anastomotic complication)) of $\geqslant 15 \%$ during the 3-6-month period following FeNO measurement. Patient No. 2 (table 1) had lacking functional data, but experienced a 
TABLE 1 Clinical data from the nine patients with unstable bronchiolitis obliterans syndrome (BOS)

\begin{tabular}{|c|c|c|c|c|c|c|c|c|c|c|c|}
\hline $\begin{array}{l}\text { Patient } \\
\text { No. }\end{array}$ & TX & $\begin{array}{c}\text { Initial } \\
\text { disease }\end{array}$ & Sex & $\begin{array}{l}\text { Age } \\
\text { yrs }\end{array}$ & Treatment $^{\#}$ & $\begin{array}{c}\text { Feno" } \\
\text { ppb }\end{array}$ & $\begin{array}{c}\text { First } \\
\text { FeNO days }^{+}\end{array}$ & $\begin{array}{l}\text { BOS } \\
\text { status }^{\#}\end{array}$ & $\begin{array}{c}\Delta \mathrm{FEV}_{1}{ }^{\S} \% \\
(\mathrm{~mL})\end{array}$ & BOS treatment ${ }^{f}$ & Clinical outcome $\# \#$ \\
\hline 2 & SL & Emp. & $\mathrm{F}$ & 47 & CsA/AZA & 26.0 & 4012 & BOS 3 & NA & & Dead, BOS 3 (9 m) \\
\hline 3 & $\mathrm{DL}$ & Emp. & M & 48 & CsA/MMF & 23.8 & 288 & BOS 0-p & $-52(-1550)$ & Pulse steroids; ATG & Dead, BOS 3 (8 m) \\
\hline 4 & SL & IPF & $\mathrm{F}$ & 42 & FK/MMF & 20.5 & 2342 & BOS 1 & $-18(-260)$ & Pulse steroids; ATG & Alive, BOS 2 (6 m) \\
\hline 6 & $\mathrm{DL}$ & Bronch. & M & 40 & CsA/AZA & 9.2 & 922 & BOS 2 & $-48(-1590)$ & Pulse steroids; ATG; switch to CsA/FK & Dead, BOS 3 (5 m) \\
\hline 7 & SL & IPF & M & 57 & CsA/AZA & 17.2 & 759 & BOS 0-p & $-16(-370)$ & Pulse steroids; switch to CsA/FK & Alive, BOS 1 (8 m) \\
\hline 8 & $\mathrm{DL}$ & IPF & $\mathrm{F}$ & 31 & CsA/AZA & 14.1 & 2079 & BOS 1 & $-29(-480)$ & Pulse steroids; ATG/MTX & Alive, BOS 2 (14 m) \\
\hline 9 & $\mathrm{SL}$ & Emp. & M & 67 & FK/AZA & 29.0 & 3342 & BOS 1 & $-16(-310)$ & Pulse steroids; ATG & Alive, BOS $2(15 \mathrm{~m})$ \\
\hline
\end{tabular}

TX: transplantation; FeNO: exhaled nitric oxide fraction; $\triangle \mathrm{FEV}$ : change in forced expiratory volume in one second (FEV1); SL: single lung; DL: double lung (bilateral); Emp.: emphysema; IPF: idiopathic pulmonary fibrosis; Bronch.: bronchiectasis; M: male; F: female; FK: tacrolimus; MMF: mycophenolate mofetil; CsA: cyclosporine A; AZA: azathioprine; BOS 0-p: potential BOS; $\geqslant$ BOS 1: established BOS; NA: not available; ATG: antithymocyte globulin; MTX: methotrexate; m: months. \#: on day of FeNO measurement; ${ }^{\prime}$ : final value; ${ }^{+}$: post-transplantation; ${ }^{\varsigma}$ : irreversible decline within 3 weeks before and 6 months after first $F$ eNo measurement expressed as percentage decrease from the highest previous baseline FEV1 (absolute decline); ${ }^{f}$ : administered within the 2 months following FeNo measurement; $\# \#$ : at the last follow-up after first FeNO measurement.

continuous functional decline attributed to the progression of BOS before death with confirmed obliterative bronchiolitis on autopsy and was, therefore, included in this group.

Analyses of FeNO measurements from recipients who exhibited an active infection were excluded owing to the known effect of infections on FeNO [9]. An active infection was diagnosed on the basis of the following: 1) clinical, radiological and/or physiological deterioration; 2) isolation of an organism from a clinically relevant BAL fluid culture; 3) antibiotic therapy resulting in a full recovery and return to pre-morbid lung function; and 4) other concurrent diagnoses excluded by TBB when appropriate. Simple colonisation was diagnosed on the basis of the following: 1) light growth of microorganisms on BAL fluid culture; 2) in the case of stable lung function, the patient did not receive any new antimicrobial therapy, and there were no adverse sequelae; and 3) in the case of deteriorating lung function, a concurrent diagnosis such as acute rejection, lymphocytic bronchiolitis or unstable BOS was established, and antibiotic therapy, if administered, had no effect on the deteriorating lung function.

Analysis of FeNO measurements from patients with reversible graft dysfunction (acute rejection or lymphocytic bronchiolitis) at the date of measurement was also excluded. For those patients with active infection or reversible graft dysfunction, no FeNO measurement was performed within the 3 months following the acute event.

For comparison, FeNO measurements performed using the same standardised method were also recorded in 21 healthy nonsmoking control volunteers without a history of asthma or allergic rhinitis and free of upper and lower respiratory symptoms on the day of the test.

\section{Exhaled nitric oxide fraction measurement}

$\mathrm{NO}$ was measured using a rapid linear-response chemiluminescent analyser (280B; Sievers, Boulder, CO, USA) with a lower limit of detection of $\sim 1 \mathrm{ppb}$ and a response time of $0.2 \mathrm{~s}(0$ $95 \%$ rise time). Before each measurement, a two-point calibration was performed using medical-grade NO (Air Liquide France, Limay, France) at concentrations of $0 \mathrm{ppb}$ (Nitrogen High Purity Gas; Air Liquide France) and 45 ppm. For single-breath NO measurements, seated subjects were asked to exhale slowly from total lung capacity through a narrow Teflon-coated tube and recordings were made of exhaled NO levels in parts per billion. As NO concentrations are highly flow dependent, a constant flow rate $\left(50 \mathrm{~mL} \cdot \mathrm{s}^{-1}\right)$ was maintained against a fixed resistance $[17,18]$. The patients targeted a constant positive mouth pressure of $12-13 \mathrm{cmH}_{2} \mathrm{O}$, which closed the velum to prevent nasal $\mathrm{NO}$ contamination [19]. The procedure was repeated until three technically acceptable measurements were recorded at the end of exhalation, i.e. three FeNO that agreed within $10 \%$ of the mean value. FeNO was calculated as the mean of these three values [17].

\section{Statistical analysis}

Continuous data are presented as mean $\pm S \mathrm{SD}$, and categorical data as count and proportion. The stable BOS-free group was analysed in order to establish normal values and the intraclass correlation coefficient. The confidence intervals of intraclass coefficients were determined using bootstrap resampling. Random-effect models were built to account for repeated FeNO measurements in each patient. Adjusted p-values for multiple comparisons are reported using the Scheffé correction.

\section{RESULTS}

A total of 50 lung transplant recipients attended for review, and 145 FeNO measurements were performed. Thirty-four patients (92 FeNO measurements) did not experience irreversible lung dysfunction throughout the study period (stable BOS-free patients). Sixteen patients showed irreversible lung 
dysfunction and were diagnosed with BOS. Among these, seven patients with BOS retained stable lung function throughout the study, six patients exhibited unstable BOS and three other patients were successively classified in both groups (unstable and then stable BOS) during the study period. Thus, 10 patients were analysed in the stable BOS group (19 FeNO measurements), and nine in the unstable BOS group (13 FeNO measurements).

The remaining $21 \mathrm{FeNO}$ measurements were excluded from analysis due to a diagnosis of active infection (17 FeNO measurements from 15 recipients) or reversible graft dysfunction (four FeNO measurements from two patients with A2 grade rejection and two patients with lymphocytic bronchiolitis) at the date of FeNO measurement.

BAL was performed in seven of the nine patients with unstable BOS at the time of FeNO measurement because of an initial decline in pulmonary function. In five cases, no organism was isolated. In the other two patients, simple colonisation with Pseudomonas aeruginosa (No. 6; table 1) and both P. aeruginosa and Aspergillus fumigatus (No. 1; table 1) was diagnosed.

The FeNO measurements for the different groups are shown in figure 1 . The range of $F$ eNO in the stable BOS-free patients $(\mathrm{n}=34)$ was $3.3-18.7 \mathrm{ppb}($ mean $\pm \mathrm{SD} 9.7 \pm 3.7 \mathrm{ppb})$. The reproducibility of FeNO measurements was good, with an intraclass correlation coefficient of 0.71 (95\% confidence interval (CI) 0.45-0.86) in a subgroup of 27 stable BOS-free patients with two measurements, and an intraclass correlation coefficient of $0.64(95 \%$ CI $0.39-0.78)$ in 14 stable BOS-free patients with three measurements. Mean FeNO in this stable BOS-free group was similar to that of the control group $(9.7 \pm 3.7$ versus $9.6 \pm$ $2.9 \mathrm{ppb}$, respectively; $\mathrm{p}=\mathrm{NS}$ ) and similar to that of the stable BOS group $(9.7 \pm 3.7$ versus $9.7 \pm 3.3 \mathrm{ppb}$; mean difference 0.02 (95\% CI 3.10-3.19); $\mathrm{p}=0.99)$. Compared with stable BOS-free

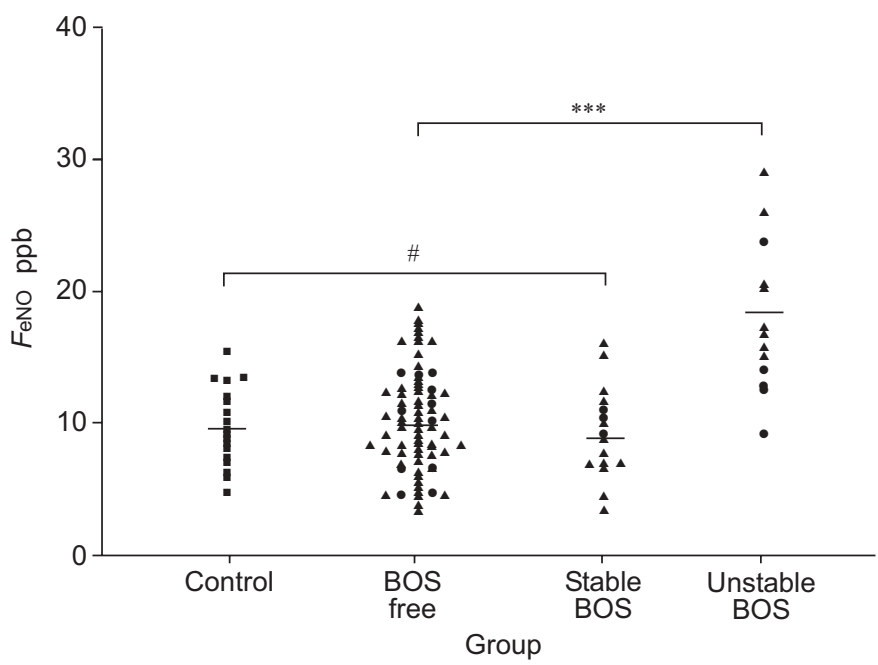

FIGURE 1. Mean exhaled nitric oxide fraction ( $F$ eNO) in lung transplant recipients with stable or unstable bronchiolitis obliterans syndrome (BOS), stable BOS-free patients and healthy controls ( $\mathbf{\square}$ : no transplantation; $\boldsymbol{\Delta}$ : single lung transplantation; $\mathbf{\bullet}$ : bilateral lung transplantation). Horizontal bars represent means. ***: $p<0.001$, unstable versus stable patients; \#: $p=0.99$ (NS), stable BOS versus BOS-free patients and controls. patients, mean values of FeNO were significantly increased in patients with unstable BOS $(9.7 \pm 3.7$ versus $18.4 \pm 5.7 \mathrm{ppb}$, respectively; mean difference 8.7 (95\% CI 6.4-11.1); $\mathrm{p}<0.001$; fig. 1). Using an FeNO of $15 \mathrm{ppb}$ as the cut-off value, the sensitivity, specificity, positive predictive value (PPV) and negative predictive value (NPV) of the mean FeNO in discriminating patients with unstable BOS from stable BOSfree patients were $77,91,70$ and $93 \%$, respectively. Three of the 34 stable BOS-free patients displayed a mean FeNO of $>15 \mathrm{ppb}$ and did not develop BOS during a follow-up of $\geqslant 9$ months.

In addition, in order to evaluate the potential bias due to the presence of a native lung on the accuracy of FeNO measurement, mean FeNO was also compared between patients who had undergone SLT and those who had undergone BLT in each diagnostic category (fig. 1). No significant difference was observed according to surgical procedure in each group $(12.9 \pm 0.67$ versus $11.7 \pm 1.10 \mathrm{ppb}$, respectively; $\mathrm{p}=0.31)$.

FeNO results from patients who had developed BOS, including patients with stable $(n=10)$ and unstable BOS $(n=9)$, are shown separately in figure 2 . The mean FeNO of patients with unstable BOS was $18.4 \pm 5.7 \mathrm{ppb}$, significantly higher than that of those with stable BOS $(9.7 \pm 3.3 \mathrm{ppb}$; mean difference 8.7 (95\% CI $6.1-9.3$ ); $<<0.001$ ). Each group (stable and unstable BOS) was subdivided according to BOS grade (BOS 1 and BOS 2/3) at the date of FeNO measurement. In both the stable and unstable BOS groups, no significant difference in FeNO was observed between patients with BOS 1 and those with BOS 2/3 (fig. 2).

The clinical data of the patients with unstable and stable BOS are detailed in tables 1 and 2. During the 6-month period following FeNO measurement, eight of the nine patients with unstable BOS displayed a fractional decrease in FEV1 of $\geqslant 15 \%$ (expressed as the percentage decrease from the highest previous baseline FEV1), with an increase in BOS stage despite

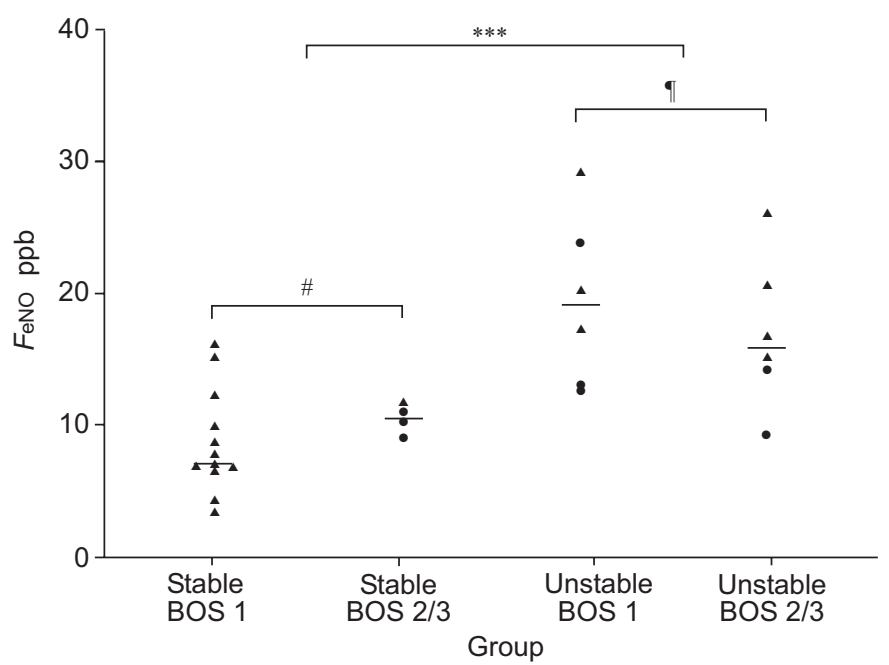

FIGURE 2. Mean exhaled nitric oxide fraction ( $F$ eNO) in lung transplant recipients with stable or unstable bronchiolitis obliterans syndrome (BOS) grade 1 or grades 2/3 ( $\boldsymbol{\Delta}$ : single lung transplantation; $\boldsymbol{0}$ : bilateral lung transplantation). Horizontal bars represent means. ${ }^{* *}: \mathrm{p}<0.001$, unstable versus stable BOS; * $\mathrm{p}=0.79$ (NS), stable BOS 1 versus stable BOS $2 / 3 ;{ }^{\natural}: p=0.84$ (NS), unstable BOS 1 versus unstable BOS $2 / 3$. 
TABLE 2 Clinical data from the 10 patients with stable bronchiolitis obliterans syndrome (BOS)

\begin{tabular}{|c|c|c|c|c|c|c|c|c|c|c|c|}
\hline $\begin{array}{l}\text { Patient } \\
\text { No. }\end{array}$ & TX & $\begin{array}{c}\text { Initial } \\
\text { disease }\end{array}$ & Sex & $\begin{array}{l}\text { Age } \\
\text { yrs }\end{array}$ & Treatment $^{\#}$ & $\begin{array}{c}\text { Feno } \\
\mathrm{ppb}\end{array}$ & $\begin{array}{c}\text { First } F \text { eNO } \\
\text { days }^{+}\end{array}$ & BOS status $\#$ & $\Delta \mathrm{FEV}_{1}{ }^{\S} \%$ & $\begin{array}{c}\text { Additional BOS } \\
\text { treatment }^{f}\end{array}$ & Clinical outcome \\
\hline 1 & $\mathrm{SL}$ & Emp. & M & 53 & FK/MMF & 6.6 & 1190 & BOS 1 & -3 & & Stable BOS 1 (14 m) \\
\hline 2 & $\mathrm{DL}$ & Bronch. & M & 44 & $\mathrm{CsA} / \mathrm{MMF}$ & 10.9 & 4069 & BOS 3 & -3 & & Stable BOS $3(6 \mathrm{~m})$ \\
\hline 3 & SL & $H X$ & $\mathrm{~F}$ & 32 & FK/MMF & 12.2 & 2719 & BOS 1 & +4 & & Stable BOS 1 (12 m) \\
\hline 4 & $\mathrm{SL}$ & Emp. & $\mathrm{F}$ & 58 & CsA/AZA & 9.8 & 3045 & BOS 2 & +2 & & Stable BOS 2 (12 m) \\
\hline 7 & SL & Emp. & $\mathrm{F}$ & 54 & FK/MMF & 8.4 & 1211 & BOS 1 & +3 & & Stable BOS $1(14 \mathrm{~m})$ \\
\hline 8 & SL & Emp. & M & 67 & FK/MMF & 10.7 & 3522 & BOS 2 & -2 & & Stable BOS $2(7 \mathrm{~m})$ \\
\hline 9 & $\mathrm{SL}$ & IPF & M & 57 & FK/MMF & 4.3 & 939 & BOS 1 & -4 & & Stable BOS 1 (4 m) \\
\hline 10 & $\mathrm{DL}$ & IPF & $\mathrm{F}$ & 31 & CsA/AZA & 10.3 & 2259 & BOS 2 & -2 & & Stable BOS $2(7 \mathrm{~m})$ \\
\hline
\end{tabular}

TX: transplantation; FeNO: exhaled nitric oxide fraction; $\Delta \mathrm{FEV}$ 1: change in forced expiratory volume in one second (FEV1); SL: single lung; DL: double lung (bilateral); Emp.: emphysema; Bronch.: bronchiectasis; HX: histiocytosis X; IPF: idiopathic pulmonary fibrosis; M: male; F: female; FK: tacrolimus; MMF: mycophenolate mofetil; CsA: cyclosporine A; AZA: azathioprine; $\geqslant$ BOS 1: established BOS; m: months. \#: on day of FeNO measurement; ": final value; ${ }^{+}$: post-transplantation; ${ }^{\S}$ : irreversible decline within 3 weeks before and 6 months after first $F_{\text {eNO }}$ measurement expressed as percentage decrease from the highest previous baseline $\mathrm{FEV}_{1}{ }^{f}:$ administered within the study period following FeNO measurement; \#\#: at the last follow-up after the first $F_{\mathrm{eNO}}$ measurement.

alteration of immunosuppression with pulse steroids, antithymocyte globulin and/or cyclosporin A/tacrolimus conversion in the vast majority of patients. In the final patient, already in BOS 3, progressive respiratory failure was observed, with ultimate death due to the progression of BOS. By contrast, patients classified as having stable BOS retained a stable FEV1 within the 6 months following FeNO measurement and did not require alteration of the immunosuppressive regimen during this period.

Taking an FeNO of $15 \mathrm{ppb}$ as the cut-off value, the sensitivity, specificity, PPV and NPV of the mean FeNO for detecting unstable BOS in each patient with BOS were 77, 100, 100 and $83 \%$, respectively. Using the same cut-off value, the sensitivity, specificity, PPV and NPV of each FeNO measurement taken independently for detecting unstable BOS were 69, 89, 81 and $80 \%$, respectively (32 FeNO measurements among 19 patients classified as having stable or unstable BOS).

There was no significant correlation between $F$ eNO and prednisone daily dose $(r=-0.18 ; p=0.2)$, serum cyclosporin A concentration $(\mathrm{r}=-0.2 ; \mathrm{p}=0.1)$ or tacrolimus trough level $(\mathrm{r}=-0.01 ; \mathrm{p}=0.95)$. The mean daily dose of steroid in the patients of each group was not significantly different among the three groups $(11.7 \pm 7.4$ (group 1), $8.6 \pm 3.0$ (group 2) and $9.3 \pm 5.9$ (group 3 ) $\mathrm{mg} \cdot \mathrm{day}^{-1} ; \mathrm{p}=\mathrm{NS}$ ).

\section{DISCUSSION}

The clinical utility of FeNO measurements in predicting functional outcome in the follow-up of lung transplant recipients with BOS was assessed. It was first observed that FeNO measurements were reproducible in a BOS-free patient with stable pulmonary function over time, owing to a high intraclass correlation coefficient. Hence, measurements of FeNO showed little day-to-day variability when subjects were clinically stable. Compared with stable BOS-free patients, elevated values of $F$ eNO were observed in patients with unstable
BOS. Using an FeNO of $15 \mathrm{ppb}$ as the cut-off value, the mean FeNO showed relatively good specificity (91\%) and NPV (93\%) for discriminating patients with unstable BOS from stable BOSfree patients; however, there were still three false-positive patients among 34 stable BOS-free patients. These three patients did not develop BOS during follow-up ( $\geqslant 9$ months), were free of respiratory symptoms and had no history of asthma. The present authors can offer no clear explanation for the high $F$ eNO in these three stable BOS-free patients and can only consider that they represent overlap levels.

Among patients with BOS, elevated values of FeNO were observed only in the case of unstable BOS, affecting patients who experienced a decline in lung function in the months following FeNO measurement. By contrast, recipients with stable BOS showed similar FeNO measurements to stable BOSfree recipients. This suggests that FeNO measurements may provide information that is useful in discriminating patients with unstable BOS from those with stable BOS. In addition, the elevated $\mathrm{FeNO}$ in patients with unstable BOS were found in both those with early disease (BOS 1) and those with more advanced disease (BOS 2/3). Conversely, the lack of elevated FeNO in patients with stable BOS was observed independently of BOS stage (BOS 1 or BOS 2/3). In other clinical lung transplantation studies, raised $F$ eNO values were found in patients with BOS $[12,13,15,20]$, and, interestingly, FeNO tended to be higher in those with recent clinical deterioration $[13,15,20]$ or in patients with early disease, i.e. BOS 1 [12]. In prospective studies of lung transplant recipients free of BOS on inclusion, VERLEDEN and coworkers [15, 20] found increased $F$ eNO values in patients who developed BOS (BOS 0 -p or BOS 1), with a decline in FEV1 during follow-up. In a cross-sectional study of lung transplant recipients, FeNO values were increased in the BOS group as a whole, but there was a trend suggesting that the highest levels were found in patients showing recent clinical deterioration [13]. Another 
cross-sectional study found an increase in FeNO in patients who had early disease (BOS 1 ), but this increase was lost in the more advanced stages of the disease, i.e. BOS 2/3 [12]. The present findings are in accordance with results from studies showing raised $F$ eNO in lung transplant recipients at the time at which they experienced an irreversible decline in lung function, including patients who are developing BOS [15, 20] or with unstable BOS [13]. Conversely, the present data are in contrast with the study of FISHER et al. [12], since the raised $F$ eNO in patients with unstable BOS were observed independently of BOS stage in the present study. In the study of FISHER et al. [12], patients were not classified as having stable or unstable BOS, and this fact could account for these conflicting results. In order to explain the observation of raised FeNO only in patients with unstable BOS among those with BOS, it can be hypothesised that successive periods of inflammatory activity occur over time in the airways of patients with BOS, leading to increased iNOS expression and a subsequent increase in exhaled NO levels, which may be downregulated when the inflammatory phase is replaced by fibrosis. This could reflect the different functional patterns already described in these patients [3]. Indeed, rapid decline in FEV1 can be followed by spontaneous or therapeutically mediated stabilisation of lung function during the course of BOS [3]. In this regard, there is no marker that can predict the different functional patterns of BOS over time. The present findings suggest that FeNO may be helpful in both precluding overt immunosuppression in patients with stable BOS and predicting early subsequent loss of lung function in those with unstable BOS, requiring early alteration of immunosuppression in order to hamper the progression of BOS. However, the question arises as to what a physician should do in clinical practice when a previously stable patient with BOS exhibits increased FeNO. Increased FeNO values are also observed in patients with respiratory infections [9], and conflicting data have been reported in lung transplant recipients showing acute rejection $[12,13,21]$. For these reasons, it seems prudent to first exclude such causes of reversible graft failure before attempting to appropriately interpret FeNO.

Measurement of FeNO in SLT recipients may have introduced some bias into the present results, since it can be hypothesised that the native lung has an impact on the measurement of FeNO after SLT. Nevertheless, it has recently been demonstrated that there is no significant difference in FeNO between SLT and BLT recipients, either in the stable condition or during chronic rejection [14]. This suggests that the impact of the native lung is negligible. The present results are concordant with these data, since the transplant procedure (SLT versus BLT) did not affect the FeNO, suggesting that, despite this potential concern, the present results are valid.

Since iNOS is very sensitive to corticosteroids, the daily dose of steroid is also a potential source of bias at the time of FeNO measurement. Nevertheless, no significant difference was found in the mean daily dose of steroid among the different groups, and no correlation between FeNO and prednisone dose was observed.

Although FeNO is also increased during infectious episodes, this seems irrelevant in the present study, due to the exclusion of every FeNO measurement from patients with an active infection at the time of measurement [9].
In summary, the present findings suggest that, in lung transplant recipients with established bronchiolitis obliterans syndrome or even potential bronchiolitis obliterans syndrome, raised exhaled nitric oxide fractions seem to indicate the development of worrisome functional impairment during follow-up. Although further studies are required, measurements of exhaled nitric oxide fraction, as a noninvasive marker, may represent a new tool for guiding immunosuppressant therapy in patients with bronchiolitis obliterans syndrome.

\section{REFERENCES}

1 Sundaresan S, Trulock EP, Mohanakumar T, Cooper JD, Patterson GA. Prevalence and outcome of bronchiolitis obliterans syndrome after lung transplantation. Washington University Lung Transplant Group. Ann Thorac Surg 1995; 60: 1341-1346.

2 Heng D, Sharples LD, McNeil K, Stewart S, Wreghitt T, Wallwork J. Bronchiolitis obliterans syndrome: incidence, natural history, prognosis, and risk factors. J Heart Lung Transplant 1998; 17: 1255-1263.

3 Nathan SD, Ross DJ, Belman MJ, et al. Bronchiolitis obliterans in single-lung transplant recipients. Chest 1995; 107: 967-972.

4 Estenne M, Maurer JR, Boehler A, et al. Bronchiolitis obliterans syndrome 2001: an update of the diagnostic criteria. J Heart Lung Transplant 2002; 21: 297-310.

5 Barnes PJ. Nitric oxide and airway disease. Ann Med 1995; 27: 389-393.

6 Gabbay E, Haydn Walters E, Orsida B, et al. In stable lung transplant recipients, exhaled nitric oxide levels positively correlate with airway neutrophilia and bronchial epithelial iNOS. Am J Respir Crit Care Med 1999; 160: 2093-2099.

7 Kharitonov SA, Chung KF, Evans D, O'Connor BJ, Barnes PJ. Increased exhaled nitric oxide in asthma is mainly derived from the lower respiratory tract. Am J Respir Crit Care Med 1996; 153: 1773-1780.

8 Kharitonov SA, Yates D, Robbins RA, Logan-Sinclair R, Shinebourne EA, Barnes PJ. Increased nitric oxide in exhaled air of asthmatic patients. Lancet 1994; 343: 133-135.

9 Kharitonov SA, Yates D, Barnes PJ. Increased nitric oxide in exhaled air of normal human subjects with upper respiratory tract infections. Eur Respir J 1995; 8: 295-297.

10 Paredi P, Kharitonov SA, Loukides S, Pantelidis P, du Bois RM, Barnes PJ. Exhaled nitric oxide is increased in active fibrosing alveolitis. Chest 1999; 115: 1352-1356.

11 Moodley YP, Lalloo UG. Exhaled nitric oxide is elevated in patients with progressive systemic sclerosis without interstitial lung disease. Chest 2001; 119: 1449-1454.

12 Fisher AJ, Gabbay E, Small T, Doig S, Dark JH, Corris PA. Cross sectional study of exhaled nitric oxide levels following lung transplantation. Thorax 1998; 53: 454-458.

13 Gabbay E, Walters EH, Orsida B, et al. Post-lung transplant bronchiolitis obliterans syndrome (BOS) is characterized by increased exhaled nitric oxide levels and epithelial inducible nitric oxide synthetase. Am J Respir Crit Care Med 2000; 162: 2182-2187.

14 Verleden GM, Dupont LJ, Delcroix M, et al. Exhaled nitric oxide after lung transplantation: impact of the native lung. Eur Respir J 2003; 21: 429-432. 
15 Verleden GM, Dupont LJ, Delcroix M, et al. Accuracy of exhaled nitric oxide (FENO) for the diagnosis of chronic rejection (CR) after lung transplantation (LTX). J Heart Lung Transplant 2003; 22: Suppl. 1S, S182.

16 Yousem SA, Berry GJ, Cagle PT, et al. Revision of the 1990 working formulation for the classification of pulmonary allograft rejection: Lung Rejection Study Group. J Heart Lung Transplant 1996; 15: 1-15.

17 American Thoracic Society. Recommendations for standardized procedures for the online and offline measurement of exhaled lower respiratory nitric oxide and nasal nitric oxide in adults and children - 1999. Am J Respir Crit Care Med 1999; 160: 2104-2117.
18 Silkoff P, McLean P, Slusky A, et al. Effect of pressure and flow on measurement of exhaled and nasal nitric oxide. Am J Respir Crit Care Med 1997; 155: 260-267.

19 Kharitonov SA, Barnes PJ. There is no nasal contribution to exhaled nitric oxide during exhalation against resistance or during breath-hold. Thorax 1997; 52: 540-544.

20 Verleden GM, Dupont L, Lamont J, et al. Is there a role for measuring exhaled nitric oxide in lung transplant recipients with chronic rejection? J Heart Lung Transplant 1998; 17: 231-232.

21 Silkoff PE, Caramori M, Tremblay L, et al. Exhaled nitric oxide in human lung transplantation. A noninvasive marker of acute lung rejection. Am J Respir Crit Care Med 1998; 157: 1822-1828. 